\title{
Effects of Preoperative Gabapentin on Postoperative Nausea and Vomiting after Open Cholecystectomy: A Prospective Randomized Double-Blind Placebo-Controlled Study
}

\author{
Saeed Khademi Fariborz Ghaffarpasand Hamid Reza Heiran Arshak Asefi
}

Department of Anesthesiology, Fasa University of Medical Sciences, Fasa, Iran

\section{Key Words}

Gabapentin · Postoperative nausea and vomiting • Open cholecystectomy

\begin{abstract}
Objective: To evaluate the effect of gabapentin on the incidence and severity of postoperative nausea and vomiting (PONV) after open cholecystectomy. Subjects and Methods: A total of 90 patients scheduled for elective open cholecystectomy were randomly assigned to either a gabapentin group (600 mg, $2 \mathrm{~h}$ before surgery) or a placebo group. For the analysis, 1 patient was excluded from the gabapentin group and 2 patients from the placebo group. A standard technique was used for anesthesia. Pethidine and metoclopramide were used for postoperative management of pain and nausea/vomiting, respectively. The prevalence of PONV, its severity (measured on visual analogue scale, VAS), and total pethidine and metoclopramide use in the first $24 \mathrm{~h}$ after the operation were recorded. Results: There were no demographic differences between the two groups. Of the 44 patients given gabapentin, $16(36.6 \%)$ and 28 of $43(65.2 \%)$ placebo patients developed PONV; the difference was statistically significant $(p=0.02)$. However, there was no difference in the severity of PONV between the gabapentin and placebo groups $(p=0.12)$. Gabapentin patients used less pethidine $(28.33 \pm 129 \mathrm{mg})$ and metoclopramide $(6.0 \pm$
\end{abstract}

\section{KARGER}

Fax +4161306 1234

E-Mail karger@karger.ch

www.karger.com
(C) 2009 S. Karger AG, Basel

1011-7571/10/0191-0057\$26.00/0

Accessible online at:

www.karger.com/mpp
$6.3 \mathrm{mg}$ ) than the placebo group $(35.1 \pm 15.1$ and $9.33 \pm 7.1$ $\mathrm{mg}$, respectively). The differences were statistically significant (pethidine: $p=0.002$, metoclopramide: $p=0.033$ ). However, gabapentin did not reduce postoperative pain significantly ( $p=0.096)$. Conclusion: Our data show that gabapentin not only reduced PONV after open cholecystectomy, but also reduced the need for additional postoperative analgesics.

Copyright $\odot 2009$ S. Karger AG, Basel

\section{Introduction}

Postoperative nausea and vomiting (PONV) are common complications following anesthesia and surgery. The etiology of PONV is complex and dependent on a variety of factors, including the technique of anesthesia, patient demographics, and type and site of surgery [1].

Gabapentin (brand name Neurontin) is a $\gamma$-aminobutyric acid (GABA) analogue. Gabapentin was originally approved in the USA by the Food and Drug Administration in 1994 for use as an adjunctive medication to control partial seizures (effective when added to other antiseizure drugs). In 2002, an indication was added for treating post-herpetic neuralgia (neuropathic pain following shingles), other painful neuropathies, and nerve-related pain [2]. Its exact mechanism of action is unknown, but 
its therapeutic action on neuropathic pain is thought to involve voltage-gated $\mathrm{N}$-type calcium ion channels. It is thought to bind to the $\alpha 2 \delta$ subunit (1 and 2) [3] of the voltage-dependent calcium channel in the central nervous system [4].

Guttuso et al. [5] demonstrated in an open clinical study the antiemetic effect of gabapentin in chemotherapy-induced acute (within $24 \mathrm{~h}$ ) and delayed onset (days 2-5) nausea and vomiting in breast cancer. Pandey et al. [1] showed that gabapentin successfully reduced PONV after laparoscopic cholecystectomy. In the present clinical study, we evaluated the antiemetic effect of gabapentin on the incidence and severity of PONV in patients who underwent open cholecystectomy.

\section{Subjects and Methods}

\section{Patients}

This study was a double-blind placebo-controlled prospective clinical trial. We enrolled 90 American Society of Anesthesiologists physical status I and II patients of both sexes who were scheduled for elective open cholecystectomy. This study was approved by the Institutional Review Board of the Fasa University of Medical Sciences. All the patients filled in the informed consent form. Based on 0.9 power to detect a significant difference ( $\mathrm{p}=0.05$, two-sided), 40 patients were required for each study group. To compensate for non-evaluable patients, we planned to enroll 45 patients per group. Patients with a BMI $>30$; a history of previous severe PONV; a history of motion sickness; significant gastrointestinal problems; recent antiemetic drug use; who were older than 60 years or younger than 18 years; who had impaired kidney or liver functions; who were menstruating, pregnant or lactating females; or who were smokers were excluded from the study. Overall, 158 patients were screened for the study, of whom 90 patients finished.

\section{Study Protocol}

At the first visit, each patient was given a sealed envelope containing their admission number in the order of referral. This was performed by a nurse who was blinded to the study. Using medical chart numbers, the patients were divided into two groups: the gabapentin group ( $n=45$; odd numbers) and the placebo group ( $\mathrm{n}=45$; even numbers). One patient from the gabapentin group and two from the placebo group did not follow through with the study, and hence were not included in the analysis.

All patients were visited for preanesthetic assessment and to explain the study protocol the day before the surgery. Patients enrolled in the gabapentin group received $600 \mathrm{mg}$ (two $300 \mathrm{mg}$ tablets), while those in the placebo group received two placebo (capsules similar in appearance to gabapentin). All the patients received $1 \mathrm{~g}$ ceftriaxone $30 \mathrm{~min}$ before surgery as antibiotic prophylaxis. All the patients underwent the same anesthetic protocol. The patients received midazolam $(0.01 \mathrm{mg} / \mathrm{kg})$ and morphine $(0.1 \mathrm{mg} / \mathrm{kg})$ as premedication. Anesthesia was induced in the patients with thiopental sodium $(3-5 \mathrm{mg} / \mathrm{kg})$ and atracurium $(0.4$
Table 1. Demographic characteristics of the patients

\begin{tabular}{lccl}
\hline & $\begin{array}{c}\text { Gabapentin } \\
(\mathrm{n}=44)\end{array}$ & $\begin{array}{c}\text { Placebo } \\
(\mathrm{n}=43)\end{array}$ & $\begin{array}{l}\mathrm{p} \\
\text { value }\end{array}$ \\
\hline Females & $41(93.2)$ & $39(90.7)$ & 0.091 \\
Males & $3(6.8)$ & $4(9.3)$ & 0.076 \\
Age, years & $51.3 \pm 16.7$ & $52.1 \pm 13.6$ & 0.868 \\
BMI & $23.4 \pm 3.04$ & $23.5 \pm 2.85$ & 0.902 \\
MAP, mm Hg & $95.8 \pm 7.93$ & $93.2 \pm 6.28$ & 0.995 \\
IV fluid intake, ml & $1,066.6 \pm 28.7$ & $1,146.6 \pm 43.4$ & 0.136 \\
Duration of surgery, min & $39.6 \pm 5.1$ & $38.8 \pm 4.9$ & 0.601 \\
\hline
\end{tabular}

Figures in parentheses are percentages. MAP $=$ Mean arterial pressure.

$\mathrm{mg} / \mathrm{kg}$ ). After 2-3 min of mask ventilation and reassurance of muscle relaxation, endotracheal intubation was carried out. During the maintenance of anesthesia, patients received a 50:50 combination of oxygen and nitrous oxide, and also isoflurane 1.5$2.5 \%$. After completion of surgery, neuromuscular blockade was reversed with neostigmine $(2.5 \mathrm{mg})$ and atropine $(1.25 \mathrm{mg} / \mathrm{kg})$. Subcostal open cholecystectomy was performed in all the patients by the same surgeon (M.H.H.), who was blinded to the study. Patients were extubated when adequate spontaneous ventilation was established, and were transferred to recovery unit. After approximately $1 \mathrm{~h}$ in the recovery unit, patients were transferred to their respective wards.

The presence of PONV, the number of episodes and its severity were recorded by a physician who was blinded to the study. Severity of patients' pain and nausea were measured quantitatively using a $10-\mathrm{cm}$ linear visual analogue scale (VAS) at 1, 4, 6, 12,18 and $24 \mathrm{~h}$ after the surgery. Pethidine $(0.5 \mathrm{mg} / \mathrm{kg})$ was given intravenously to patients who had a pain score more than 4 . $\mathrm{Pa}$ tients who had a VAS score more than 4 in nausea also received metoclopramide $(10 \mathrm{mg}$ ) intravenously.

Statistical Analysis

The Statistical Package for Social Science, SPSS for Windows, version 15.0 (SPSS, Chicago, Ill., USA) was used for data analysis. Paired t tests were used to compare results within groups; independent $t$ tests were used to compare results between the groups; $\chi^{2}$ tests were used to compare proportions. Data are reported as means \pm SD for $95 \%$ CI with $5 \%$ degree of freedom. A 2 -sided $p$ value $<0.05$ was considered statistically significant.

\section{Results}

There were no demographic differences (gender distribution, age, BMI, duration of surgery, mean arterial pressure and IV fluid intake during the surgery) between the 2 study groups (table 1 ). In the first $24 \mathrm{~h}$ after open cholecystectomy, 16 (36.6\%) patients in the gabapentin 
Table 2. Incidence of PONV and its severity

\begin{tabular}{lccc}
\hline & $\begin{array}{l}\text { Gabapentin } \\
(\mathrm{n}=44)\end{array}$ & $\begin{array}{l}\text { Placebo } \\
(\mathrm{n}=43)\end{array}$ & $\begin{array}{l}\mathrm{p} \\
\text { value }\end{array}$ \\
\hline PONV incidence & $16(36.6 \%)$ & $28(65.2 \%)$ & 0.021 \\
Severity of PONV & $4.4 \pm 2.13$ & $5.6 \pm 1.95$ & 0.119 \\
Postoperative pain & $4.46 \pm 0.83$ & $5.13 \pm 1.24$ & 0.096 \\
Metoclopramide use, mg & $6.0 \pm 6.3$ & $9.33 \pm 7.1$ & 0.033 \\
Pethidine use, mg & $28.33 \pm 12.9$ & $35.1 \pm 15.1$ & 0.002 \\
\hline
\end{tabular}

group and 28 (65.2\%) in the placebo group developed PONV ( $p=0.021)$. There was no significant difference in the severity of PONV between the two groups (table 2). However, the metoclopramide use for control of PONV was significantly lower $(\mathrm{p}=0.033)$ in the gabapentin group $(6.0 \pm 6.3 \mathrm{mg})$ compared to the control group $(9.33$ $\pm 7.1 \mathrm{mg})$. The mean VAS scores for postoperative pain in the gabapentin and placebo groups were $4.46 \pm 0.83$ and $5.13 \pm 1.24$, respectively, and the difference was not statistically significant $(\mathrm{p}=0.096)$. However, patients in the placebo group used significantly more pethidine compared to the patients in the gabapentin group (35.1 \pm 15.1 vs. $28.33 \pm 12.9 \mathrm{mg} ; \mathrm{p}=0.002$ ) for pain relief (table 2).

\section{Discussion}

Our study showed that gabapentin significantly reduced the incidence of PONV after open cholecystectomy surgery, but it did not decrease the severity of PONV. It also reduced the need for postoperative pethidine and metoclopramide administration for the management of pain and PONV, thus indicating that gabapentin possess antiemetic and analgesic properties. However, it did not reduce postoperative pain, as measured by the VAS.

It has been reported that one third of patients undergoing general anesthesia suffer from PONV [6]. Although more than 1,000 investigations on the prevention or treatment of PONV have been published, the incidence of PONV has not changed over the past two decades [7]. The etiology of PONV following open cholecystectomy remains unclear, but it is probably associated with operative factors. Previous studies $[8,9]$ have shown that female gender, longer anesthesia time, general anesthesia, not smoking, use of postoperative opioids and previous PONV and/or motion sickness were associated with in- creased incidence and severity of PONV. Although less important, PONV may also be influenced by the type of surgery as well: strabismus correction and laparoscopic surgery especially have been described as risk factors for PONV $[10,11]$. Most of the previous studies that addressed the effect of gabapentin on reducing PONV incidence and severity have been performed on laparoscopic surgery procedures $[1,12]$.

Our study confirmed previous studies $[1,13]$ involving the use of gabapentin in patients undergoing laparoscopic cholecystectomy. Pandey et al. [1] in a randomized double-blind placebo-controlled study showed that preoperative administration of $600 \mathrm{mg}$ gabapentin significantly reduced the incidence of PONV in patients undergoing laparoscopic cholecystectomy (46/125 vs. 75/125; $\mathrm{p}=0.04)$. It also reduced postoperative fentanyl use for pain control. However, it did not have any significant effect on the severity of PONV. In another study, performed by Mohammadi and Seyedi [13], it was shown that 300 mg gabapentin given preoperatively significantly reduced the severity of PONV and the need for additional analgesic after laparoscopic surgery for assisted reproductive technologies, but it did not reduce the incidence of PONV.

Apparently, gabapentin has also been shown to reduce the incidence of nausea and vomiting after chemotherapy by a postulated mechanism of mitigation of tachykinin neurotransmitter activity [14]. The etiology of PONV in patients undergoing open cholecystectomy is not identical to that in patients receiving cytotoxic drugs, but we assume that it may be one probable mechanism in the prevention of PONV by gabapentin. As all the variables were similar between study groups, the difference in the incidence of PONV could only be attributed to gabapentin.

\section{Conclusion}

Our data show that gabapentin not only reduced PONV after open cholecystectomy, but also reduced the need for additional postoperative analgesics.

\section{Acknowledgment}

The authors wish to thank all the patients who participated in the study and their families. 


\section{References}

1 Pandey CK, Priye S, Ambesh SP, Singh S, Singh U, Singh PK: Prophylactic gabapentin for prevention of postoperative nausea and vomiting in patients undergoing laparoscopic cholecystectomy: a randomized, doubleblind, placebo-controlled study. J Postgrad Med 2006;52:97-100.

$\checkmark 2$ Goa KL, Sorkin EM: Gabapentin: a review of its pharmacological properties and clinical potential in epilepsy. Drugs 1993;46:409427.

3 Hendrich J, Van Minh AT, Heblich F, NietoRostro M, Watschinger K, Striessnig J, et al: Pharmacological disruption of calcium channel trafficking by the alpha2delta ligand gabapentin. Proc Natl Acad Sci USA 2008; 105:3628-3633.

-4 Davies A, Hendrich J, Van Minh AT, Wratten J, Douglas L, Dolphin AC: Functional biology of the alpha(2)delta subunits of voltage-gated calcium channels. Trends Pharmacol Sci 2007;28:220-228.

5 Guttuso T Jr, Roscoe J, Griggs J: Effect of gabapentin on nausea induced by chemotherapy in patients with breast cancer. Lancet 2003;361:1703-1705.
-6 Apfel CC, Korttila K, Abdalla M, Kerger H, Turan A, Vedder I, Zernak C, Danner K, Jokela R, Pocock SJ, Trenkler S, Kredel M, Biedler A, Sessler DI, Roewer N, IMPACT Investigators: A factorial trial of six interventions for the prevention of postoperative nausea and vomiting. N Engl J Med 2004; 350:2441-2451.

7 White PF: Prevention of postoperative nausea and vomiting - a multimodal solution to a persistent problem. N Engl J Med 2004; 350:2511-2512.

8 Sinclair DR, Chung F, Mezei G: Can postoperative nausea and vomiting be predicted? Anesthesiology 1999;91:109-118.

-9 Apfel CC, Laara E, Koivuranta M, Greim CA, Roewer N: A simplified risk score for predicting postoperative nausea and vomiting: conclusions from cross-validations between two centers. Anesthesiology 1999;91: 693-700.
10 Piper SN, Suttner SW, Röhm KD, Maleck WH, Larbig E, Boldt J: Dolasetron, but not metoclopramide prevents nausea and vomiting in patients undergoing laparoscopic cholecystectomy. Can J Anaesth 2002;49:1021-1028.

11 Apfel CC, Greim CA, Haubitz I, Grundt D, Goepfert C, Sefrin P, Roewer N: The discriminating power of a risk score for postoperative vomiting in adults undergoing various types of surgery. Acta Anaesthesiol Scand 1998;42:502-509.

12 Pandey CK, Priye S, Singh S, Singh U, Singh RB, Singh PK: Preemptive use of gabapentin significantly decreases postoperative pain and rescue analgesic requirements in laparoscopic cholecystectomy. Can J Anaesth 2004; 51:358-363.

13 Mohammadi SS, Seyedi M: Effects of gabapentin on early postoperative pain, nausea and vomiting in laparoscopic surgery for assisted reproductive technologies. Pak J Biol Sci 2008;11:1878-1880.

- 14 Guttuso T Jr, Kurlan R, McDermott MP, Kieburtz K: Gabapentin's effects on hot flushes in postmenopausal women: a randomized controlled trial. Obstet Gynecol 2003;101: 337-345. 